\title{
The doctor as patient: better informed but similar in behaviour
}

- Cite as: CMAJ 2019 August 19;191:E928-9. doi: 10.1503/cmaj.109-5802

Posted on cmajnews.com on August 1, 2019.

$\mathrm{T}$ here are few people, if any, better informed about health care than physicians. Yet when physicians find themselves on the other end of the stethoscope, as patients, the choices they make about their own health care don't appear to differ much from that of other patients.

A recent paper - "Is Great Information Good Enough? Evidence from Physicians as Patients" - by the National Bureau of Economic Research in the United States looked at 10 years of data from the Military Health System. The database includes claims data for military physicians who receive medical care.

The researchers wanted to know if physicians - as intelligent, well-informed patients - pursued care for themselves that was proven to be of "high value" and avoided care that evidence suggests has less clinical merit. The answer, they found, was not really.

The results suggest that simply providing more information to patients is unlikely to improve health outcomes, according to one of the paper's coauthors, Dr. Anupam Jena, a general medicine physician and economist in the department of health care policy at Harvard Medical School.

"The cornerstone of public health is the belief that if you inform patients about risks - of smoking, of not exercising, of drinking excessively - that you can change behaviour, that information and education is the key," said Jena. "If that was true, then the most sophisticated, most informed consumer of knowledge would always choose the right care. We did not observe that. What we showed is that doctors have the same difficulties patients do."
It's widely accepted, for example, that there's little benefit to preoperative diagnostic testing for low-risk surgeries, such as a chest $x$-ray before eye surgery. But the preoperative testing rate in such scenarios was the same for both physicians and "regular" patients (35\%). Similarly, despite World Health Organization guidelines sug- value. Doctors were no more likely to follow diabetes care guidelines, for instance, or to take statins for heart disease. The researchers did find slightly higher rates of medication adherence for physicians recently diagnosed with hypertension or hypercholesterolemia, but the differences were modest.

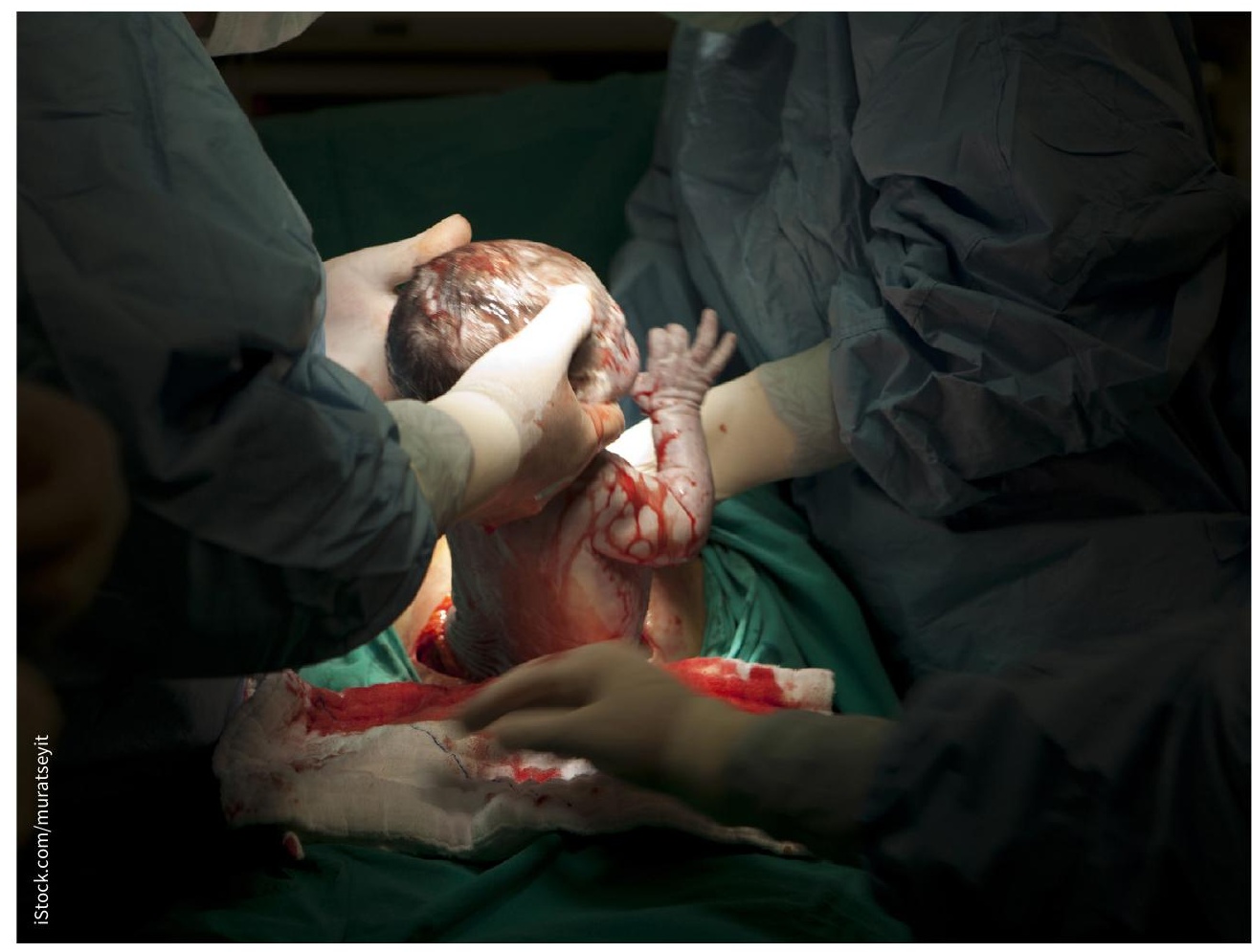

The cesarean rate among physician and nonphysician patients was similar in a recent analysis of US military data.

gesting only $10 \%-15 \%$ of women require cesarean section, the researchers found that $29 \%$ of physicians giving birth had the procedure, a rate only slightly below that of other patients (31\%).

Physicians and other patients also had similar adherence rates to some forms of care considered to be of high
One lesson physicians can learn from this paper, said Jena, is that providing information to a patient about their health and treatments is just one step in providing them with appropriate care. "It will likely require additional steps, such as checking in with them more regularly, or having a nurse call back with a reminder, 
or perhaps an electronic reminder linked to their medical record."

David Blackburn, a research chair in patient adherence to drug therapy at the University of Saskatchewan, isn't surprised by the findings of the paper. Knowledge is but one of many factors driving treatment adherence, so providing more information will not, on its own, yield better uptake. According to Blackburn, the health care system needs to do more to support patients and to identify and address concerns they have about the care prescribed for them.
"At the end of the day, they (physicians) are just another patient," said Blackburn. "Everyone is the same. We all have our own concerns, and attitudes, and biases, depending on our experiences and perspectives."

There may be one unique factor, however, keeping physicians from following the advice of other doctors: a lingering attitude in medical culture of toughing it out when you have health problems. But the increased attention being paid to physician wellness and burnout in recent years is starting to reduce the stigma attached to asking for help, according to Dr. Lyle Mittelsteadt, assistant executive director of professional affairs for the Alberta Medical Association.

"In Alberta, we are seeing more and more physicians seeking assistance," said Mittelsteadt, who is responsible for the association's Physician and Family Support Program and is senior staff lead for the province's Well Doc initiative. "Physicians are realizing it's okay to ask for help. We see that as a positive."

Greg Basky, Saskatoon, Sask. 